\title{
RESPOSTA DA ALFAFA A FONTES DE FÓSFORO ASSOCIADAS AO GESSO E À CALAGEM ${ }^{1}$
}

\author{
Patrícia Sarmento²*; Moacyr Corsi $;$; Fábio Prudêncio de Campos ${ }^{4}$ \\ ${ }^{2}$ Pós-Graduando do Depto. de Produção Animal - USP/ESALQ. \\ ${ }^{3}$ Depto. de Produção Animal - USP/ESALQ, C.P. 9 - CEP: 13418-900 - Piracicaba, SP. \\ ${ }^{4}$ Pós-Graduando do Depto. de Zootecnia - FCAV/UNESP, Rod. Carlos Tonani, Km 5 - CEP: 14870-000 - \\ Jaboticabal, SP.
}

\begin{abstract}
RESUMO: O fósforo é um dos nutriente mais importante na produção de alfafa nos solos brasileiros. Diversas fontes de $\mathrm{P}$ são disponíveis no mercado e o fosfato de Gafsa (FG) é considerado tão eficiente como solúvel. A eficiência dos adubos fosfatados é afetada pela acidez do solo. O uso do gesso associado ao FG pode corrigir o perfil do solo em relação ao alumínio e diminuir a fixação do $P$ no solo. Portanto, o objetivo deste trabalho foi avaliar num experimento em vasos a eficiência do superfosfato triplo (ST), do FG e do FG com gesso, aplicados antes e depois da calagem, nas doses de 50, 100 e $200 \mathrm{mg} \mathrm{P} \mathrm{dm}^{-3}$. Foi utilizado um solo classificado como LATOSSOLO VERMELHO-AMARELO Alumínico. Foram efetuados três plantios de alfafa, realizando-se um corte no primeiro plantio e três cortes no terceiro. Com o ST obteve-se maior produção de matéria seca (MS) $(3,3 \mathrm{~g} / \mathrm{vaso})$ do que com o FG (1,0 g/vaso) no primeiro plantio. Mas no terceiro plantio ocorreu menor produção de MS com o uso do ST (2,4 g/vaso) do que com o FG (6,0 g/vaso). O gesso com FG elevou a produção de MS (7,0 g/vaso) em relação ao FG (3,7 g/vaso) no terceiro plantio. A aplicação do ST depois da calagem aumentou a produção de MS (5,0 g/vaso) comparado a aplicação antes da calagem (3,7 $\mathrm{g}$ (vaso), no primeiro plantio. Não houve efeito do momento de calagem para o FG com ou sem gesso.

Palavras-chaves: Medicago sativa, fosfato de Gafsa, superfosfato triplo, gesso, calagem
\end{abstract}

\section{ALFALFA RESPONSE TO PHOSPHORUS SOURCES ASSOCIATED TO GYPSUM AND LIMING}

\begin{abstract}
ABSTRAT: Phosphorus is one of the most important nutrients in alfalfa production in Brazilian soils. Several P sources are available in the market and the Gafsa phosphate (GP) is considered as efficient and soluble. The efficiency of phosphate fertilizers is affected by soil acidity. The use of GP associated to gypsum can aliviate Al toxicity and reduce soil phosphorus fixation. Therefore, this study was curried out in a glasshouse experiment, aiming to evaluate the efficiency of the triple superphosphate (TS) and GP and GP with gypsum, applied before and after liming at the rates of 50; 100 and $200 \mathrm{mg} \mathrm{P} \mathrm{dm}^{-3}$. The soil was classified as a Typic Hapludox. Alfalfa was seeded three times and plants were harvested only once in the first seeding and three times in the third seeding. In the first seeding alfalfa yield (DM) was higher (3.3 g/pot) under TS than GP (1.0 g/pot), but in the third seeding TS produced less $(2.4 \mathrm{~g} / \mathrm{pot})$ than GP (6.0 g/pot). GP with gypsum with increased DM yield $(7.0 \mathrm{~g} / \mathrm{pot})$ in relation to GP $(3.7 \mathrm{~g} / \mathrm{pot})$ in the third seeding. Alfalfa DM was higher under TS applied after liming ( $5.0 \mathrm{~g} / \mathrm{pot})$ than when TS was applied before liming, in the first seeding (3.7 g/pot). GP with or without gypsum presented similar response in terms of DM yield when applied before or after liming.

Key words: Medicago sativa L., Gafsa phosphate, triple superphosphate, gypsum, liming
\end{abstract}

\section{INTRODUÇÃO}

Derivado de diferentes fontes, o fósforo $(P)$ é um nutriente mineral que apresenta-se em várias formas químicas. Assim, diversos adubos fosfatados são encontrados no comércio, distinguindo-se na concentração de P e na sua solubilidade (Raij, 1991).

Devido ao baixo nível de $P$ nos solos brasileiros, a longevidade da cultura da alfafa e a produção são dependentes da adubação fosfatada para 0 estabelecimento e manutenção do estande. Dessa maneira, os altos preços dos adubos fosfatados impulsionam as pesquisas para conseguir maneiras mais eficientes e econômicas de se combinar a produção da alfafa com as fontes fosfatadas disponíveis. Os fosfatos solúveis em água, são as melhores fontes de adubos fosfatados, mas, são os mais caros (Raij et al., 1992). Existem os fosfatos de rocha nacionais que são de menor preço, mas por serem de baixa eficiência agronômica em relação às fontes solúveis, não são aceitos pelos produtores. Por outro lado, os fosfatos naturais importados, como o de Gafsa, que possuem maior solubilidade que os nacionais, têm apresentado eficiência semelhante a dos fosfatos solúveis (Raij et al., 1992), mostrando ser competitivos com os mesmos, tornando o FG uma opção interessante como fonte de $P$ para a produção de alfafa.

Os fosfatos solúveis em água passam rapidamente para a fase sólida (lábil) e com o passar do tempo são adsorvidos pelo solo (não lábil), tornado

${ }^{1}$ Parte da Dissertação de Mestrado do primeiro autor apresentada à USP/ESALQ - Piracicaba, SP. 
indisponível para a planta. No entanto, o aumento do $\mathrm{pH}$ do solo para 5,5 a 6,5 melhora sua eficiência devido à diminuição da fixação do $P$ (Goedert \& Sousa, 1984).

Os fosfatos naturais aumentam sua eficiência com o passar do tempo devido ao acréscimo em sua solubilização (Goedert \& Sousa, 1984), mas necessitam de certa acidez e contato com o solo para que ocorra a solubilização do mesmo sugerindo que, para sua utilização deve haver incorporação ao solo e certo período de incubação (Magalhães et al., 1987). Em pH menor que 5,5 ocorre a fixação do $\mathrm{P}$ pelo alumínio (Goedert \& Sousa, 1984), sendo que a utilização de gesso junto à adubação fosfatada poderia ser uma alternativa para diminuir o teor de alumínio no perfil do solo e, conseqüentemente, decrescer a fixação do $P$ sem interferir com a acidez do solo necessária à solubilização do FG.

Considerando que os fertilizantes fosfatados são essenciais no aumento da produtividade da alfafa e que seu uso está relacionado a maior custo de produção das explorações agrícolas, torna-se necessário aprimorar técnicas e conhecimentos para aumentar a eficiência desses fertilizantes. $O$ uso do gesso com o FG pode corrigir o perfil do solo em relação ao alumínio, diminuir a fixação do $P$ e conseqüentemente aumentar a eficiência do adubo. Uma vez que, a eficiência dos adubos fosfatados é afetada pela acidez do solo, o uso da calagem não deve ser desconsiderado nas pesquisas envolvendo fontes de fósforo.

O objetivo deste trabalho foi avaliar a produção de MS da alfafa, além do teor de $\mathrm{P}$ e o pH no solo, com o uso do $\mathrm{FG}$ em relação ao ST, utilização do gesso junto da aplicação do FG e realizado antes e após a aplicação de calagem, em função das doses de P; bem como de obter informações sobre essas variáveis em função de doses de fontes de P. A pesquisa visa, também, obter 0 efeito dos tratamentos com fontes e doses de $P$, no decorrer dos cortes de alfafa.

\section{MATERIAL E MÉTODOS}

O experimento foi conduzido em estufa da ESALQ (Escola Superior de Agricultura "Luiz de Queiroz"), localizado no município de Piracicaba - SP. O solo utilizado foi coletado do Instituto de Zootecnia de Nova Odessa (SP), apresentava textura média arenosa e foi classificado como LATOSSOLO VERMELHOAMARELO Alumínico. O solo apresentava as seguintes características químicas: $\mathrm{pH}=4,1 ; \mathrm{MO}=18,6 \mathrm{mg} \mathrm{dm}^{-3} ; \mathrm{P}$ (resina) $=3,0 \mathrm{~g} \mathrm{dm}^{-3} ; \mathrm{S}^{-\mathrm{SO}_{4}}=31,9 \mathrm{~g} \mathrm{dm}^{-3} ; \mathrm{K}=0,5 \mathrm{mmolc}$ $\mathrm{dm}^{-3} ; \mathrm{Ca}=6,0 \mathrm{mmolc} \mathrm{dm}^{-3} ; \mathrm{Mg}=3,0 \mathrm{mmolc} \mathrm{dm}^{-3} ; \mathrm{Al}=$ $9,0 \mathrm{mmolc} \mathrm{dm}^{-3} ; \mathrm{H}+\mathrm{Al}=38,1 \mathrm{mmolc} \mathrm{dm}^{-3} ; \mathrm{T}=47,6$ mmolc $\mathrm{dm}^{-3}$ e V $=20,0 \%$.

Os tratamentos foram: superfosfato triplo aplicado antes da calagem (STAC); superfosfato triplo depois da calagem (STDC); fosfato de Gafsa antes da calagem (FGAC); fosfato de Gafsa depois da calagem
(FGDC); fosfato de Gafsa associado ao gesso antes da calagem (FGGAC) e fosfato de Gafsa associado ao gesso depois da calagem (FGGDC). Foram utilizadas as doses de $0,50,100$ e $200 \mathrm{mg} \mathrm{P} \mathrm{dm}^{-3}$. O superfosfato triplo e o fosfato de Gafsa apresentaram 47,11 e 27,39\% de $\mathrm{P}_{2} \mathrm{O}_{5}$ total e 40,02 e $11,06 \%$ de $\mathrm{P}_{2} \mathrm{O}_{5}$ solúvel em ácido cítrico, respectivamente.

Realizaram-se três plantios (05/10/97, 25/01/98 e 12/04/98) devido ao ataque de fungos Fusarium, nas raízes das plantas. As sementes da alfafa cultivar Crioula (primeiro plantio) e cv. XAI 32 (terceiro plantio) foram inoculadas com Rhizobium meliloti SEMIA 116. No primeiro plantio, a alfafa foi colhida com 47 dias. $O$ segundo plantio não foi avaliado. No terceiro plantio, o primeiro corte da alfafa foi realizado com três meses de idade (10/07/98), e os dois restantes foram feitos a cada 30 dias (09/08/98 e 08/09/98, respectivamente), a 7,5 cm acima da superfície do solo.

As adubações com micronutrientes e $\mathrm{KCl}$ foram realizadas juntamente com a primeira semeadura (TABELA 1). As adubações de manutenção foram realizadas após cada corte que consistiram na aplicação de $\mathrm{KCl}$, de acordo com o teor de $\mathrm{K}$ na $\mathrm{MS}$ que foi considerado como sendo 3,6\%. Assim, após a determinação da produção de MS por tratamento calculou-se a adubação potássica. A calagem foi realizada 60 dias antes ou 60 após a aplicação dos adubos fosfatados, visando elevar a saturação por bases a $85 \%$, utilizando-se calcário dolomítico. Quando utilizou-se gesso (1/3 do Ca adicionado como calcário) junto ao $F G$ a dose de calcário adicionada foi $2 / 3$ do utilizado para o tratamento que não recebeu gesso. Aplicou-se enxofre elementar nos tratamentos que não receberam gesso para manter o equilíbrio no fornecimento de enxofre.

A irrigação dos vasos, com água de torneira, foi realizada para manter a capacidade de campo do solo em $70 \%$. Os vasos foram pesados diariamente para completar a água utilizada pelo sistema solo-planta.

Foi avaliado a produção de MS da parte aérea da alfafa, em um corte no primeiro plantio e três cortes no terceiro plantio e o peso de MS das raízes das plantas do terceiro plantio mediante lavagem com água corrente. O material, tanto da parte aérea quanto da raiz, foi seco em estufa a $65^{\circ} \mathrm{C}$ até peso constante.

$\mathrm{O} \mathrm{pH}$ e as concentrações de $\mathrm{P}$ no solo foram medidas em amostras tiradas antes do terceiro plantio e após a última colheita. A disponibilidade de $\mathrm{P}$ no solo foi medida pelo método de resina trocadora de íons (Raij et al., 1986).

O delineamento estatístico utilizado em blocos completos ao acaso com três repetições para estudo dos resultados relativos à produção de MS da parte aérea do primeiro plantio e produção de MS das raízes das plantas no terceiro plantio. $O$ delineamento foi 0 de blocos completos ao acaso com parcelas subdivididas no tempo com três repetições para a produção de MS 
da parte aérea do terceiro plantio, $\mathrm{pH}$ e concentração de $P$ no solo. Um fator que compôs as parcelas foi representado pelos tratamentos com fontes de fósforo associadas ou não ao gesso e momento de calagem; o outro fator que compôs as parcelas foi as doses de $\mathrm{P}$ e, as subparcelas foram representadas pelos cortes ou momentos de amostragem do solo.

Os dados de produção de MS da parte aérea e da raiz, ambos do terceiro plantio, foram analisados segundo a transformação quadrática e os dados de concentração de $\mathrm{P}$ no solo segundo a transformação logarítima.

TABELA 1 - Composição das soluções usadas no ensaio em vaso.

\begin{tabular}{lccc}
\hline Produto & Elemento & $\begin{array}{c}\text { Concentração } \\
\text { do elemento }\end{array}$ & $\begin{array}{c}\text { Concentração } \\
\text { do produto }\end{array}$ \\
\hline $\mathrm{KCl}$ & $\mathrm{K}$ & 171 & $17,41^{(1)}$ \\
$\mathrm{mg} \mathrm{dm}_{3} \mathrm{BO}_{3}$ & $\mathrm{~B}$ & 1,0 & $0,96^{(2)}$ \\
$\mathrm{CoCl}_{2} \cdot 2 \mathrm{H}_{2} \mathrm{O}$ & $\mathrm{Co}$ & 0,1 & $0,05^{(2)}$ \\
$\mathrm{CuSO}_{4} \cdot 5 \mathrm{H}_{2} \mathrm{O}$ & $\mathrm{Cu}$ & 1,5 & $1,88^{(2)}$ \\
$\mathrm{FeSO}_{4} \cdot 7 \mathrm{H}_{\mathrm{O}}$ & $\mathrm{Fe}$ & 5 & $4,30^{(2)}$ \\
$\mathrm{MnSO}_{4} \cdot \mathrm{H}_{2} \mathrm{O}$ & $\mathrm{Mn}$ & 5 & $3,14^{(2)}$ \\
$\mathrm{NaMo}_{\mathrm{ZnSO}} \cdot 7 \mathrm{H}_{2} \mathrm{O}$ & $\mathrm{Mo}$ & 0,1 & $0,04^{(2)}$ \\
$\mathrm{Zn}_{2}$ & 5 & $4,08^{(2)}$ \\
\hline
\end{tabular}

(1)Utilizou-se $100 \mathrm{~mL}$ de solução por vaso de $6 \mathrm{~kg}$ de solo aplicado em uma única dose antes do primeiro plantio.

(2)Utilizou-se $30 \mathrm{~mL}$ de solução por vaso de $6 \mathrm{~kg}$ de solo aplicado em uma única dose antes do primeiro plantio.
Os resultdos foram submetidos à análise de variância através do programa estatístico SANEST (Sarriés et al., 1993). Foi utilizado o teste $t$ (contrastes ortogonais) na comparação entre os tratamentos com fontes de $P$ para produção de MS da alfafa e concentração de $\mathrm{P}$ no solo, e o teste de Tukey para o $\mathrm{pH}$ do solo. Em relação às doses de $\mathrm{P}$ utilizou-se a análise de regressão e para os cortes e momentos de amostragem do solo o teste de Tukey.

\section{RESULTADOS E DISCUSSÃO}

\section{Produção de matéria seca}

Os resultados experimentais do segundo plantio não foram analisados, pois o ataque de fungo do gênero Fusarium comprometeu completamente as produções de MS. Após a autoclavagem do solo, procedeu-se um terceiro plantio. Para efeito deste trabalho, são apresentados os resultados das análises do primeiro (um corte) e terceiro plantios (três cortes).

A alfafa não persistiu nos vasos sob a dose zero de $P$ nos dois plantios, seja por problemas nutricionais (terceiro plantio) ou por se tornarem, as plantas, mais suscetível ao fungo (primeiro plantio). Em função disto optou-se por eliminar tal tratamento da análise da variância.

A produção média de MS da parte aérea da alfafa em decorrência dos tratamentos com fontes e doses de $P$, obtido no primeiro e terceiro plantio, está apresentado na TABELA 2. Ocorreram interações $(P<0,05)$ entre os tratamentos com fontes de $P$ e doses,

TABELA 2 - Produções de matéria seca da parte aérea de alfafa obtidos em todos os tratamentos com fontes e doses de $\mathrm{P}$ referente ao primeiro e terceiro plantios.

\begin{tabular}{|c|c|c|c|c|c|c|}
\hline \multirow[b]{2}{*}{ Doses de P } & \multicolumn{6}{|c|}{ Tratamentos com fontes de P } \\
\hline & STAC $^{(1)}$ & $\operatorname{STDC}^{(2)}$ & $\mathrm{FGAC}^{(3)}$ & FGD $^{(4)}$ & FGGAC $^{(5)}$ & FGGD C $^{(6)}$ \\
\hline$--m^{\prime} d^{-3}$ & \multicolumn{6}{|c|}{ 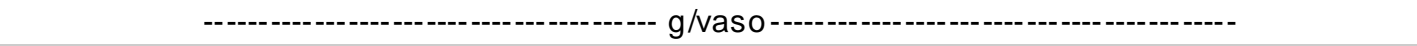 } \\
\hline \multicolumn{7}{|c|}{ Primeiro plantio } \\
\hline 50 & 0,90 & 1,35 & 0,35 & 0,34 & 0,45 & 0,44 \\
\hline 100 & 2,75 & 4,29 & 1,45 & 1,23 & 0,86 & 0,60 \\
\hline 200 & 4,71 & 5,71 & 1,92 & 1,91 & 1,24 & 1,64 \\
\hline \multicolumn{7}{|c|}{ Terceiro plantio } \\
\hline & \multicolumn{6}{|c|}{ Corte 1} \\
\hline 50 & $0,08 a^{(7)}$ & 0,08 a & $0,13 \mathrm{a}$ & $0,15 \mathrm{~b}$ & $0,17 \mathrm{a}$ & $0,89 \mathrm{~b}$ \\
\hline 100 & $0,27 \mathrm{~b}$ & $1,69 \mathrm{~b}$ & $2,77 \mathrm{~b}$ & $4,14 \mathrm{~b}$ & $3,38 \mathrm{~b}$ & 8,15 a \\
\hline 200 & $11,93 a b$ & $14,47 \mathrm{a}$ & $9,80 \mathrm{a}$ & $9,09 \mathrm{a}$ & $17,47 \mathrm{a}$ & $16,38 \mathrm{a}$ \\
\hline & \multicolumn{6}{|c|}{ Corte 2} \\
\hline 50 & $0,06 \mathrm{a}$ & $0,05 \mathrm{a}$ & $0,10 a$ & $0,93 a b$ & $0,40 a$ & $3,25 \mathrm{a}$ \\
\hline 100 & $0,86 a b$ & $3,36 a$ & $7,76 \mathrm{a}$ & 9,53 a & $9,02 \mathrm{a}$ & 11,03 a \\
\hline 200 & $12,63 \mathrm{a}$ & $12,53 a b$ & $12,00 \mathrm{a}$ & $12,91 \mathrm{a}$ & $14,95 \mathrm{a}$ & $16,47 \mathrm{a}$ \\
\hline & \multicolumn{6}{|c|}{ Corte 3} \\
\hline 50 & $0,03 \mathrm{a}$ & $0,02 \mathrm{a}$ & $0,08 \mathrm{a}$ & $1,90 \mathrm{a}$ & $0,91 \mathrm{a}$ & $3,57 \mathrm{a}$ \\
\hline 100 & $1,35 a$ & $3,79 a$ & $7,29 \mathrm{a}$ & $8,58 \mathrm{a}$ & $8,11 \mathrm{a}$ & $8,00 \mathrm{a}$ \\
\hline 200 & $8,42 \mathrm{~b}$ & $8,75 \mathrm{~b}$ & $12,32 \mathrm{a}$ & $11,21 \mathrm{a}$ & $10,46 \mathrm{~b}$ & $11,71 \mathrm{~b}$ \\
\hline
\end{tabular}


no primeiro plantio, e entre fontes, doses e cortes, no terceiro plantio.

Houve aumento significativo de produção de MS da alfafa $(P<0,05)$ devido à adubação fosfatada no primeiro plantio (TABELA 2). Efeito favorável da adição de $P$ na produção de alfafa foi encontrado também por Yupanqui (1997) e Moreira (1997). O melhor ajuste no modelo matemático para explicar a produção de MS em função das doses de $\mathrm{P}$, foi o quadrático para o tratamento STDC e linear para os demais, no primeiro plantio (TABELA 4).

No primeiro plantio, ocorreu maior resposta da alfafa $(P<0,05)$ à adubação com $S T$ do que com $F G$ (TABELA 2 e contraste 1 da TABELA 3) em todas as doses de P. Isso deve-se a maior disponibilidade de $P$ proveniente da fonte solúvel e o fato do fosfato de rocha apresentar baixa solubilidade e, conseqüentemente, lenta liberação de P para as plantas (Novais et al., 1990). Esses resultados confirmam as observações realizadas por Moreira (1997).

O ST aplicado depois da calagem (STDC) beneficiou $(P<0,05)$ a eficiência desse adubo nas doses de 100 e $200 \mathrm{mg} \mathrm{P} \mathrm{dm}^{-3}$ (TABELA 2 e contraste 2 da TABELA 3) uma vez que o uso do calcário pode ter reduzido o teor de Al trocável no solo, diminuindo a capacidade de fixação do $\mathrm{P}$ pelo $\mathrm{Al}^{3+}$ (Goedert \& Sousa, 1984).

Não foi observada diferença estatística $(P>0,05)$ entre a calagem feita antes ou depois da aplicação do FG com ou sem gesso, no primeiro plantio (TABELA 2 e contrastes 4 e 5 da TABELA 3), como também, não houve efeito $(P<0,05)$ do $F G$ com gesso (TABELA 2 e contraste 3 da TABELA 3) comparado ao FG.

No terceiro plantio, a produção de MS aumentou $(\mathrm{P}<0,05)$ com o aumento da dose de 50 para $200 \mathrm{mg} \mathrm{P} \mathrm{dm}^{-3}$ nos três cortes (TABELA 4). Em geral, o ST apresentou melhor ajuste para o modelo linear devido à menor produção de MS da parte aérea da alfafa em decorrência, provavelmente, da fixação do $\mathrm{P}$ pelo solo por causa do atraso na realização do terceiro plantio. Na TABELA 4 está calculada a dose de $P$ para cada fonte que poderia expressar a máxima produção de MS da alfafa. Esses cálculos só puderam ser realizados para os tratamentos que apresentavam equações polinomial do segundo grau. Yupanqui (1997) afirma que as doses de $P$ recomendáveis para proporcionar produção máxima refletem as características químicas dos solos, principalmente a capacidade tampão dos fosfatos e a disponibilidade de $\mathrm{P}$ dos solos.

TABELA 3 - Contrastes ortogonais para comparação da produção de matéria seca da parte aérea de alfafa entre os tratamentos com fontes de fósforo dentro de cada dose de $\mathrm{P}$, no primeiro e terceiro plantios.

\begin{tabular}{|c|c|c|c|c|}
\hline & \multirow{2}{*}{ Contrastes } & \multicolumn{3}{|c|}{ Doses de $\mathrm{P}\left(\mathrm{mg} \mathrm{dm}^{-3}\right)$} \\
\hline & & 50 & 100 & 200 \\
\hline № & & \multicolumn{3}{|c|}{ Valor do contraste } \\
\hline \multicolumn{5}{|c|}{ Primeiro plantio } \\
\hline 1 & $2 S T A C+2 S T D C-F G A C-F G D C-F G G A C-F G G D C$ & $3,88^{*}$ & $10,07^{*}$ & $15,28^{*}$ \\
\hline 2 & STAC - STDC & $-0,63$ & $-1,68^{*}$ & $-1,23^{*}$ \\
\hline 3 & $F G A C+F G D C-F G G A C-F G G D C$ & $-0,01$ & 1,22 & 0,94 \\
\hline 4 & FGAC - FGDC & $-0,04$ & 0,31 & 0,003 \\
\hline 5 & FGGAC - FGGDC & $-0,01$ & 0,26 & $-0,41$ \\
\hline \multicolumn{5}{|c|}{ Terceiro plantio } \\
\hline \multicolumn{5}{|c|}{ Corte 1} \\
\hline 6 & $2 S T A C+2 S T D C-F G A C-F G D C-F G G A C-F G G D C$ & $-0,96$ & $-5,15^{*}$ & 0,01 \\
\hline 7 & STAC - STDC & $-0,005$ & $-0,61$ & $-0,40$ \\
\hline 8 & FGAC + FGDC - FGGAC - FGGDC & $-0,61$ & $-0,95$ & $-2,04^{*}$ \\
\hline 9 & FGAC - FGDC & $-0,02$ & $-0,39$ & $-0,01$ \\
\hline 10 & FGGAC - FGGDC & $-0,53$ & $-1,04^{*}$ & 0,15 \\
\hline \multicolumn{5}{|c|}{ Corte 2} \\
\hline 11 & $2 S T A C+2 S T D C-F G A C-F G D C-F G G A C-F G G D C$ & $-2,65^{*}$ & $-6,93^{*}$ & $-0,91$ \\
\hline 12 & STAC - STDC & 0,01 & $-0,92^{*}$ & 0,01 \\
\hline & FGAC + FGDC - FGGAC - FGGDC & $-1,23^{*}$ & $-0,46$ & $-0,78$ \\
\hline 14 & FGAC - FGDC & $-0,58$ & $-0,31$ & $-0,24$ \\
\hline 15 & FGGAC - FGGDC & $-1,18^{*}$ & $-0,33$ & $-0,19$ \\
\hline \multicolumn{5}{|c|}{ Corte 3} \\
\hline & $2 S T A C+2 S T D C-F G A C-F G D C-F G G A C-F G G D C$ & $-3,56^{*}$ & $-5,30^{*}$ & $-1,99^{*}$ \\
\hline 17 & STAC - STDC & 0,02 & $-0,81^{*}$ & $-0,05$ \\
\hline 18 & $F G A C+F G D C-F G G A C-F G G D C$ & $-1,28^{*}$ & $-0,05$ & 0,34 \\
\hline 19 & FGAC - FGDC & $-1,00^{*}$ & $-0,21$ & 0,02 \\
\hline 20 & FGGAC - FGGDC & $-1,04^{*}$ & 0,02 & $-0,18$ \\
\hline
\end{tabular}

(STAC: super triplo antes da calagem; STDC: super triplo depois da calagem; FGAC: Fosfato de Gafsa antes da calagem; FGDC: Fosfato de Gafsa depois da calagem; FGGAC: Fosfato de Gafsa com gesso antes da calagem; FGGDC: Fosfato de Gafsa com gesso depois da calagem).

${ }^{*}$ significativo a $5 \%$. 
O desempenho na produção de MS da parte aérea da alfafa, no terceiro plantio, foi maior $(P<0,05)$ com o uso do $F G$ em relação à utilização do $S T$, em todos os cortes (TABELA 2 e contrastes 6,11 e 16 da TABELA 3). No entanto, as exceções foram para as doses de $50 \mathrm{mg} \mathrm{P} \mathrm{dm}^{-3}$, no primeiro corte, e $200 \mathrm{mg} \mathrm{P}$ $\mathrm{dm}^{-3}$, no primeiro e segundo cortes, que não apresentaram diferenças $(P>0,05)$ entre esses tratamentos. (TABELA 2 e contrastes 6 e 11 da TABELA 3). A menor eficiência na utilização do ST deve-se, possivelmente, à rápida formação do $\mathrm{P}$ não lábil a partir da fonte solúvel de $\mathrm{P}$, tornando-se indisponível a planta o que é explicado por Novais et al. (1990). Por outro lado, o menor pH do solo com o uso do ST do que com FG (TABELA 8) pode ter contribuído para a redução na produção de alfafa.

No primeiro corte do terceiro plantio a produção de MS da parte aérea da alfafa foi superior $(P<0,05)$ com uso do gesso associado ao FG aplicados antes e depois da calagem (FGGAC e FGGDC) comparado ao
FGAC e FGDC na dose de $200 \mathrm{mg} \mathrm{P} \mathrm{dm}^{-3}$ (TABELA 2 e contraste 8 da TABELA 3). Isso, também, ocorreu para a dose de $50 \mathrm{mg} \mathrm{P} \mathrm{dm}^{-3}$, no segundo e terceiro cortes (TABELA 2 e contrastes 13 e 18 da TABELA 3). No entanto, esses resultados, possivelmente, são atribuídos ao aumento do $\mathrm{pH}$ do solo pelo uso do gesso (TABELA 8) e não à solubilização do FG uma vez que o nível de $P$ disponível é semelhante para o $F G$ aplicado com e sem gesso em amostragem do solo realizada antes do terceiro plantio (contraste 28 da TABELA 11). Resultado de aumento da produção de MS da alfafa com a elevação do pH do solo foi relatado por Rando (1992).

Houve maior $(\mathrm{P}<0,05)$ produção de $\mathrm{MS}$ com 0 uso do STDC comparado ao STAC, no segundo e terceiro cortes do terceiro plantio na dose de $100 \mathrm{mg} P$ $\mathrm{dm}^{-3}$ (TABELA 2 e contrastes 12 e 17 da TABELA 3). Isso é devido à calagem antes da adubação fosfatada diminuir a capacidade de adsorção ou fixação do $\mathrm{P}$ pelo solo, segundo Raij (1992).

TABELA 4 - Equações de regressão ajustadas entre produções de matéria seca da parte aérea da alfafa (Y), como variável dependente da dose de $\mathrm{P}(\mathrm{X})$, no primeiro e terceiro plantios e doses de $\mathrm{P}$ calculados para máxima produção.

\begin{tabular}{|c|c|c|c|c|}
\hline \multirow{2}{*}{$\begin{array}{l}\text { Tratamentos com } \\
\text { fontes de P }\end{array}$} & \multirow{2}{*}{ Equações } & \multirow[t]{2}{*}{$\mathrm{R}^{2}$} & \multirow{2}{*}{$\frac{\text { Dose de } \mathrm{P}^{(1)}}{\mathrm{mg} \mathrm{dm^{-3 }}}$} & \multirow{2}{*}{$\begin{array}{c}\text { Produção }^{(2)} \\
\text { g/vaso }\end{array}$} \\
\hline & & & & \\
\hline \multicolumn{5}{|l|}{ Primeiro plantio } \\
\hline STAC & $Y=0,012937+0,024873 X$ & $0,98^{*}$ & - & - \\
\hline STDC & $Y=-2,25955+0,092080 X-0,00025 X^{2}$ & $1,00^{*}$ & 184,16 & 6,22 \\
\hline FGAC & $Y=0,194175+0,0093692 X$ & $0,80^{*}$ & - & - \\
\hline FGDC & $Y=0,075981+0,0095881 X$ & $0,95^{*}$ & - & - \\
\hline FGGAC & $Y=0,278385+0,0050783 X$ & $0,93^{*}$ & - & - \\
\hline FGGDC & $Y=-0,047515+0,0083403 X$ & $0,97^{\star}$ & - & - \\
\hline \multicolumn{5}{|l|}{ Terceiro plantio } \\
\hline \multicolumn{5}{|c|}{ Corte 1} \\
\hline STAC & $Y=-5,74620+0,08435 X$ & $0,90^{*}$ & - & - \\
\hline STDC & $Y=-6,31012+0,10049 X$ & $0,95^{*}$ & - & - \\
\hline FGAC & $Y=-3,38083+0,06527 X$ & $0,99^{*}$ & - & - \\
\hline FGDC & $Y=-4,67057+0,10487 X-0,00017 X^{2}$ & $1,00^{*}$ & 313,03 & 11,49 \\
\hline FGGAC & $Y=-6,87560+0,11898 X$ & $0,98^{*}$ & - & - \\
\hline FGGDC & $Y=-8,46624+0,20821 X-0,00042 X^{2}$ & $1,00^{*}$ & 247,90 & 17,34 \\
\hline \multicolumn{5}{|c|}{ Corte 2} \\
\hline STAC & $Y=-5,82378+0,08865 X$ & $0,93^{*}$ & - & - \\
\hline STDC & $Y=-4,52983+0,08439 X$ & $0,99^{*}$ & - & - \\
\hline FGAC & $Y=-11,26883+0,26430 X-0,00074 X^{2}$ & $1,00^{*}$ & 178,65 & 12,33 \\
\hline FGDC & $Y=-12,0046+0,30228 X-0,00087 X^{2}$ & $1,00^{*}$ & 173,78 & 14,25 \\
\hline FGGAC & $Y=-11,99208+0,28551 X-0,00075 X^{2}$ & $1,00^{*}$ & 189,35 & 15,18 \\
\hline FGGDC & $Y=-7,91558+0,25703 X-0,0006815 X^{2}$ & $1,00^{*}$ & 190,23 & 16,31 \\
\hline \multicolumn{5}{|c|}{ Corte 3} \\
\hline STAC & $Y=-3,50767+0,05805 X$ & $0,96^{*}$ & - & - \\
\hline STDC & $Y=-4,60289+0,10109 X-0,00017 X^{2}$ & $1,00^{*}$ & 294,56 & 10,42 \\
\hline FGAC & $Y=-10,25889+0,23807 X-0,00063 X^{2}$ & $1,00^{*}$ & 190,24 & 12,23 \\
\hline FGDC & $Y=-7,95333+0,22900 X-0,00064 X^{2}$ & $1,00^{*}$ & 178,90 & 12,53 \\
\hline FGGAC & $Y=-10,3009+0,26440 X-0,00080 X^{2}$ & $1,00^{*}$ & 164,55 & 11,54 \\
\hline FGGDC & $Y=1,71667+0,05181 X$ & $0,94^{*}$ & - & - \\
\hline
\end{tabular}

(STAC: super triplo antes da calagem; STDC: super triplo depois da calagem; FGAC: Fosfato de Gafsa antes da calagem; FGDC: Fosfato de Gafsa depois da calagem; FGGAC: Fosfato de Gafsa com gesso antes da calagem; FGGDC: Fosfato de Gafsa com gesso depois da calagem). ${ }^{(1)}$ Dose de P para a produção máxima de MS da parte aérea estimada pela equação de regressão. ${ }^{(2)}$ Produção máxima de MS da parte aérea estimada pela equação de regressão. *significativo a $5 \%$. 
Ocorreu menor produção de $M S(P<0,05)$ quando aplicou-se o FG juntamente com gesso antes da calagem (FGGAC) comparado ao FGGDC no primeiro corte à dose de $100 \mathrm{mg} \mathrm{P} \mathrm{dm}^{-3}$ e no segundo corte à dose de $50 \mathrm{mg} \mathrm{P} \mathrm{dm}^{-3}$ (TABELA 2 e contrastes 10 e 15 da TABELA 3). O FG associado ou não ao gesso aplicado antes da calagem (FGAC e FGGAC) apresentou menor $(P<0,05)$ produção em relação ao $F G D C$ e FGGDC, no terceiro corte à dose de $50 \mathrm{mg} \mathrm{P} \mathrm{dm}^{-3}$ (contrastes 19 e 20 da TABELA 3). Isso pode ser devido à fixação do $\mathrm{P}$ ao solo, no entanto, não condiz com os resultados obtidos por Hanafi et al., (1992) que verificaram $46 \%$, em média, da dissolução do $F G$ incubado em solo ácido (pH 4,4) pelo período de 50 dias. Porém, Chu et al., (1962) afirmam que a solubilização de fosfato de rocha em certos solos ácidos depende da quantia de $\mathrm{Al}^{3+} \mathrm{e} \mathrm{Fe}^{3+}$ no sistema do solo.

$\mathrm{Na}$ utilização do $\mathrm{FG}$ antes e depois da calagem (FGAC e FGDC) e do FGGAC, as produções de MS aumentaram $(P<0,05)$ para doses de $100 \mathrm{mg} \mathrm{P} \mathrm{dm}^{-3}$, após o primeiro corte, com respostas similares no segundo e terceiro cortes (TABELA 2). Isso repetiu-se $(\mathrm{P}<0,05)$ com o uso do FGDC, na dose de $50 \mathrm{mg}$ $\mathrm{P} \mathrm{dm}{ }^{-3}$. Apesar do resultado não ser significativo observouse que na dose de $200 \mathrm{mg} \mathrm{P} \mathrm{dm}^{-3}$ também ocorreu elevação da produção de MS do primeiro para o segundo corte nos tratamentos FGAC e FGDC (TABELA 2). Esses resultados foram devidos, provavelmente, ao acréscimo da solubilização do fosfato de rocha, que estão de acordo com as observações de Moreira (1997). No entanto, ocorreu diminuição $(P<0,05)$ na produção de $M S$ no terceiro corte com o uso do FGGAC e FGGDC à dose de $200 \mathrm{mg} \mathrm{P} \mathrm{dm}^{-3}$ (TABELA 2).

As produções de MS da raiz coletadas após o último corte do terceiro plantio são apresentadas na TABELA 5 , sendo verificada a interação $(P<0,05)$ entre os tratamentos com fontes e doses de P.

Os tratamentos com ST, nas doses de 50 e 100 $\mathrm{mg} \mathrm{P} \mathrm{dm}{ }^{-3}$, apresentaram produções inferiores $(P<0,05)$ de MS de raízes em relação ao FG (TABELA 5 e contraste 21 da TABELA 6). Isso deve-se, provavelmente, à

TABELA 5 - Peso de matéria seca das raízes de alfafa obtidas em todos os tratamentos com fontes e doses de P.

\begin{tabular}{crrrrrr}
\hline & \multicolumn{6}{c}{ Tratamentos com fontes de P } \\
\cline { 2 - 7 } Doses de P & STAC & STDC & FGAC FGDC FGGAC FGGDC \\
\hline $\mathrm{mg} \mathrm{dm}^{-3}$ & \multicolumn{6}{c}{ g/vaso } \\
50 & 0,24 & 0,20 & 0,83 & 5,69 & 2,98 & 15,66 \\
100 & 5,70 & 13,58 & 25,08 & 30,07 & 29,27 & 40,29 \\
200 & 37,48 & 40,19 & 35,13 & 35,25 & 56,52 & 50,43
\end{tabular}

(STAC: super triplo antes da calagem; STDC: super triplo depois da calagem; FGAC: Fosfato de Gafsa antes da calagem; FGDC: Fosfato de Gafsa depois da calagem; FGGAC: Fosfato de Gafsa com gesso antes da calagem; FGGDC: Fosfato de Gafsa com gesso depois da calagem).

*Média de três repetições.

$\mathrm{CV}=16,87 \%$. solubilização do FG com o tempo aumentando a disponibilidade de $P$ à planta. Outro fator a ser considerado é o efeito do $\mathrm{pH}$ do solo que foi maior quando utilizou-se o FG comparado com o ST (TABELA 8).

Houve maiores $(P<0,05)$ produções de MS de raízes com a adição do ST depois da calagem comparado a sua adição antes da calagem somente na dose de 100 mg P dm${ }^{-3}$ (TABELA 5 e contraste 22 da TABELA 6). Por outro lado o FG associado ou não ao gesso depois da calagem também teve efeito benéfico $(P<0,05)$ em relação à sua utilização antes da calagem, na dose de $50 \mathrm{mg} P$ $\mathrm{dm}^{-3}$ (TABELA 5 e contrastes 24 e 25 da TABELA 6).

Em relação à utilização do $F G$ ocorreu maiores $(\mathrm{P}<0,05)$ produções de $\mathrm{MS}$ de raiz com adição de gesso às doses de 50 e $200 \mathrm{mg} \mathrm{P} \mathrm{dm}^{-3}$ (TABELA 5 e contraste 23 da TABELA 6) devido, provavelmente, ao efeito benéfico do gesso sobre o desenvolvimento das raízes.

Analisando-se os efeitos dos tratamentos com gesso foi observado que esse produto proporcionou aumento $(7,20 \%)$ do $\mathrm{pH}$ do solo em relação aos tratamentos com FG (TABELA 8). Mugwira \& Haque (1993) verificaram aumento no peso das raízes de alfafa em função da elevação do pH do solo. Sabe-se que o alumínio é o principal elemento tóxico associado à acidez do solo e sua ação se faz sentir principalmente nas raízes das plantas, prejudicando o seu crescimento (Raij, 1988). Por outro lado, o Mn também é outro elemento tóxico associado ao baixo $\mathrm{pH}$, onde a raiz é afetada de forma indireta através do resultado da inibição no crescimento da parte aérea (Smith et al., 1983).

Houve aumento linear $(P<0,05)$ de peso de raiz com as doses de $P$ aplicadas (TABELA 7) resultado que está de acordo com Mugwira \& Haque (1993). Segundo Rhykerd \& Overdahl (1972) o P ajuda as raízes a desenvolverem-se mais rapidamente melhorando a absorção de nutrientes pela planta.

\section{Valores de $\mathrm{pH}$ no solo}

Foram encontradas interações $(P<0,05)$ entre os tratamentos com fontes de $P$ e momentos de amostragem do solo e, entre momentos de amostragem e doses de $\mathrm{P}$ para $\mathrm{pH}$ do solo.

$O$ uso do $F G$ associado ou não ao gesso foram os tratamentos que apresentaram maior $(P<0,05)$ ação neutralizante no solo para os dois momentos de amostragem (TABELA 8). Isso está de acordo com as observações de Goedert \& Lobato (1984) os quais verificaram maior $\mathrm{pH}$ no solo quando foi utilizado o $\mathrm{FG}$ comparado ao ST.

Os solos que apresentam altos níveis de manganês e com pH igual ou menor que 5,5 podem induzir toxidez desse elemento na planta (KabataPendias \& Pendias, 1985) e, a cada incremento de 0,1 unidade de $\mathrm{pH}$ há uma redução de 10 vezes na atividade do íon manganês na solução do solo (Bataglia, citado por Rando, 1992). Dessa maneira é possível que os tratamentos com FG, onde o pH do solo apresentou-se maior em 0,15 unidades em relação aos tratamentos com 
TABELA 6 - Contrastes ortogonais para comparação de peso de matéria seca da raiz entre os tratamentos com fontes de fósforo dentro de cada dose de $\mathrm{P}$, no terceiro plantio.

\begin{tabular}{llccc}
\hline & \multicolumn{1}{c}{ Contrastes } & \multicolumn{2}{c}{ Doses de P $\left(\mathrm{mg} \mathrm{dm}^{-3}\right)$} \\
\cline { 3 - 5 } & & \multicolumn{3}{c}{ Valor do contraste } \\
\hline № & & $-6,62^{*}$ & $-10,26^{*}$ & $-1,62$ \\
21 & 2STAC + 2STDC - FGAC - FGDC - FGGAC - FGGDC & 0,04 & $-1,38^{\star}$ & $-0,24$ \\
22 & STAC - STDC & $-2,75^{*}$ & $-1,27$ & $-2,82^{*}$ \\
23 & FGAC + FGDC - FGGAC - FGGDC & $-1,38^{*}$ & $-0,49$ & $-0,07$ \\
24 & FGAC - FGDC & $-2,24^{*}$ & $-0,89$ & 0,41 \\
\hline
\end{tabular}

(STAC: super triplo antes da calagem; STDC: super triplo depois da calagem; FGAC: Fosfato de Gafsa antes da calagem; FGDC: Fosfato de Gafsa depois da calagem; FGGAC: Fosfato de Gafsa com gesso antes da calagem; FGGDC: Fosfato de Gafsa com gesso depois da calagem). * significativo a $5 \%$.

TABELA 7 - Equações de regressão ajustadas entre produção de matéria seca da raiz da alfafa (Y), como variável dependente da dose de $\mathrm{P}$ $(\mathrm{X})$.

\begin{tabular}{lcc}
\hline $\begin{array}{c}\text { Tratamentos com } \\
\text { fontes de } P\end{array}$ & \multicolumn{1}{c}{ Equações } & $R^{2}$ \\
\hline STAC & $Y=-15,65000+0,25818 X$ & $0,96^{*}$ \\
STDC & $Y=-13,10333+0,26652 X$ & $1,00^{*}$ \\
FGAC & $Y=-4,19833+0,21038 X$ & $0,83^{*}$ \\
FGDC & $Y=3,10333+0,17629 X$ & $0,73^{*}$ \\
FGGAC & $Y=-10,65167+0,34492 X$ & $0,97^{*}$ \\
FGGDC & $Y=10,59500+0,21313 X$ & $0,83^{*}$ \\
\hline
\end{tabular}

(STAC: super triplo antes da calagem; STDC: super triplo depois da calagem; FGAC: Fosfato de Gafsa antes da calagem; FGDC: Fosfato de Gafsa depois da calagem; FGGAC: Fosfato de Gafsa com gesso antes da calagem; FGGDC: Fosfato de Gafsa com gesso depois da calagem).

${ }^{*}$ significativo a $5 \%$.

ST, antes do terceiro plantio (TABELA 8), tenham diminuído o efeito tóxico do Mn que estava alto no solo

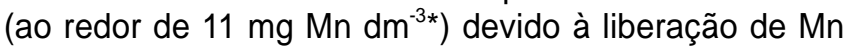
pelo processo de autoclavagem.

Dentre as leguminosas, a alfafa talvez seja a mais exigente em $\mathrm{pH}$ do solo sendo sua faixa ótima de 6,5 a 7,5 (Honda \& Honda, 1990) e a que apresenta menor tolerância ao excesso de manganês (Andrew \& Hutron, citados por Rando, 1992).

$\mathrm{O} \mathrm{pH}$ do solo que recebeu o tratamento $\mathrm{FG}+$ $G$ se manteve semelhante $(P>0,05)$ ao tratamento com FG antes do terceiro plantio (TABELA 8). Porém, na amostragem do solo, após o último corte, o pH do solo com 0 uso do $F G+G$ foi superior $(P<0,05)$ ao $F G$ (TABELA 8). Rechcigl et al., (1988) afirmaram que os íons cálcio $\left(\mathrm{Ca}^{2+}\right)$ e o sulfato $\left(\mathrm{SO}_{4}^{2-}\right)$ resultantes da hidrólise do gesso não são neutralizantes e, conseqüentemente, não diminuem a acidez do solo. Entretanto, segundo Sousa \& Ritchey (1985) o aumento de $\mathrm{pH}$ do solo, com o uso do gesso, tem sido atribuído ao domínio da reação de troca do sulfato pela hidroxila $\left(\mathrm{OH}^{-}\right)$das superfícies dos óxidos de ferro e alumínio.
TABELA 8 - Valores de $\mathrm{pH}\left(\mathrm{CaCl}_{2}\right)$ no solo antes do plantio e depois da última colheita de alfafa, nos diferentes tratamentos com fontes de P. (Médias de todas as doses de $\mathrm{P}$ aplicadas antes e após a calagem e repetições.)

\begin{tabular}{|c|c|c|c|}
\hline \multirow[b]{2}{*}{ Perío do de amostragem } & \multicolumn{3}{|c|}{$\begin{array}{l}\text { Tratamentos com fontes de } \\
\text { fósforo }\end{array}$} \\
\hline & ST & $F G$ & $\mathrm{FG}+\mathrm{G}$ \\
\hline & \multicolumn{3}{|c|}{------- Valores de pH ------ } \\
\hline Antes do terceiro plantio & $5,56 \mathrm{Ab}$ & $5,67 \mathrm{Aa}$ & $5,74 \mathrm{Ba}$ \\
\hline Após o último corte & $5,28 \mathrm{Bc}$ & $5,78 \mathrm{Ab}$ & $6,06 \mathrm{Aa}$ \\
\hline
\end{tabular}

CV $($ parcela $)=1,5 \%$.

CV (subparcela) $=2,6 \%$

*médias seguidas por letras maiúsculas distintas, numa mesma coluna e por letras minúsculas distintas, numa mesma linha, diferem a $5 \%$ pelo teste de Tukey.

Em relação às épocas de amostragens do solo, antes do terceiro plantio e depois do último corte, somente o ST apresentou redução $(\mathrm{P}<0,05)$ do $\mathrm{pH}$ do solo (TABELA 8). Isso, provavelmente, se deve à dissociação do $\mathrm{CO}_{2}$ liberado pela respiração das raízes e nódulos da alfafa (Heichel et al., 1988). Novais et al. (1990) afirmam que a acidificação da rizosfera pela planta é benéfica à solubilização do fosfato natural. No entanto a diminuição do $\mathrm{pH}$ não foi observada no presente trabalho quando se utilizou o FG associado ou não ao gesso. Ao contrário, houve elevação do $\mathrm{pH}$ do solo após o último corte com o uso do gesso (TABELA 8), por causa, talvez, à continuidade da reação do gesso e da fonte de $\mathrm{P}$ no solo.

Houve queda $(\mathrm{P}<0,05)$ do $\mathrm{pH}$ do solo após o último corte para o tratamento que empregou $50 \mathrm{mg} P$ $\mathrm{dm}^{-3}$ (TABELA 9). Isso também se deve, provavelmente, à dissociação do $\mathrm{CO}_{2}$ liberado pela respiração das raízes e nódulos da alfafa (Heichel et al., 1988). O mesmo não ocorreu entretanto nas outras doses de P (TABELA 9) devido, possivelmente, ao maior poder neutralizante presente nas elevadas doses de adubos fosfatados impedindo o abaixamento do $\mathrm{pH}$ nesse período. 
Houve aumento $(\mathrm{P}<0,05)$ no pH do solo em função das doses de $P$ (Figura 1). Isso foi, também, verificado por Smyth \& Sanchez (1980), sendo que estes autores atribuíram o resultado à liberação do $\mathrm{OH}^{-}$durante 0 processo de adsorção do P. Goedert \& Lobato (1984), utilizando o FG como fonte de $\mathrm{P}$ verificaram aumento do $\mathrm{pH}\left(\mathrm{H}_{2} \mathrm{O}\right)$ do solo de 4,9 na testemunha para 5,2 com a aplicação de $88 \mathrm{~kg} P$ $\mathrm{ha}^{-1}$, e 5,5 com o uso de $350 \mathrm{~kg} \mathrm{P} \mathrm{ha}^{-1}$.

\section{Concentração de $P$ no solo}

As concentrações de $\mathrm{P}$ no solo antes do terceiro plantio e após o último corte estão apresentadas na TABELA 10. Houve efeito $(P<0,05)$ das doses de $P$, bem como a interação $(P<0,05)$ entre momento de amostragem e os tratamentos com fontes de $P$.

Houve elevação $(P<0,05)$ na concentração de fósforo no solo com as doses de $P$ aplicadas de diferentes fontes. A equação de regressão que melhor ajustou-se aos dados foi: $Y=3,35+0,126 X, r^{2}=0,99$. Resultados semelhantes foram encontrados por Moreira (1997).

Antes do terceiro plantio, as concentrações de $P$ no solo foram maiores $(P<0,05)$ com a utilização do ST do que com o uso do FG (TABELA 10 e contraste 26 da TABELA 11). Esse resultado está de acordo com os obtidos por Goedert \& Lobato (1984), no entanto parece contraditório, uma vez que a produção de MS da parte aérea foi maior com o uso do $F G$ em relação ao ST, no terceiro plantio (TABELA 2). Dessa maneira, isso pode estar relacionado ao aumento do $\mathrm{pH}$ do solo com a utilização do FG (TABELA 8).

$\mathrm{Na}$ amostragem do solo após o último corte, os tratamentos com $\mathrm{FG}$ apresentaram teores mais elevados de $P$ no solo $(P<0,05)$ que o ST (TABELA 10 e contraste 26 da TABELA 11). Esse resultado foi atribuído ao aumento $(P<0,05)$ do $P$ no solo com o uso do $F G$ após o último corte (TABELA 12), devido a solubilização do FG com o tempo, confirmando as afirmações de Goedert \& Sousa (1984).

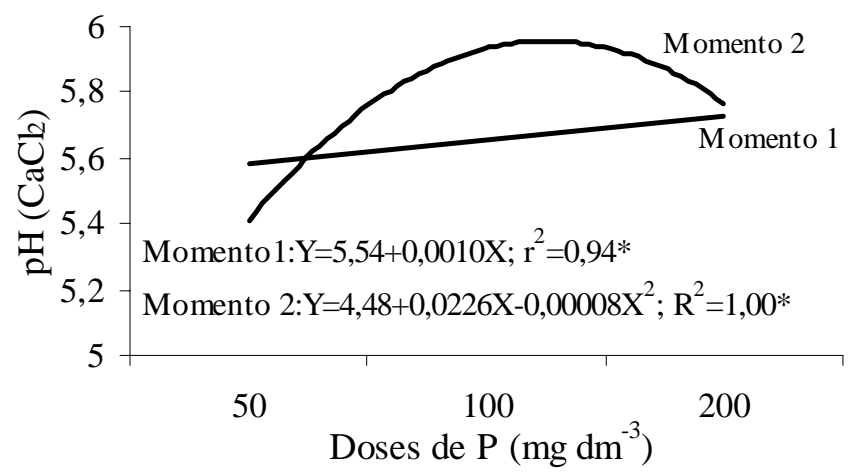

Figura 1 - $\mathrm{pH}$ no solo $\left(\mathrm{CaCl}_{2}\right)$ conforme as doses de $\mathrm{P}$ nos diferentes momentos de amostragem do solo. (Média de três repetições e 6 tratamentos com fontes de P).

TABELA 9 - Valores de $\mathrm{pH}\left(\mathrm{CaCl}_{2}\right)$ no solo antes do terceiro plantio e depois da última colheita de alfafa, conforme as doses de P. (Médias de todas as doses de $\mathrm{P}$ aplicadas antes e após a calagem e repetições).

\begin{tabular}{lccc}
\hline \multirow{2}{*}{ Momento de amostragem } & \multicolumn{3}{c}{ Doses de $\mathrm{P}\left(\mathrm{mg} \mathrm{dm}^{-3}\right)$} \\
\cline { 2 - 4 } & 50 & 100 & 200 \\
\hline & ------ Valores de pH $---\cdots--$ \\
Antes do terceiro plantio & 5,58 a & 5,66 b & 5,77 a \\
Após o último corte & 5,41 b & 5,93 a & 5,73 a
\end{tabular}

(ST: média do super triplo aplicados antes e após a calagem; FG: média do Fosfato de Gafsa aplicados antes e após a calagem; FG+G: média do Fosfato de Gafsa mais gesso aplicados antes e após a calagem).

CV (subparcela) $=2,6 \%$

*médias seguidas por letras distintas, numa mesma coluna diferem a $5 \%$ pelo teste de Tukey.

TABELA 10 - Teor de P no solo antes do terceiro plantio e após o último corte, em função dos tratamentos com fontes e doses de $P$.

\begin{tabular}{|c|c|c|c|c|c|c|}
\hline \multirow[b]{2}{*}{ Doses de $\mathrm{P}$} & \multicolumn{6}{|c|}{ Tratamentos com fontes de $\mathrm{P}$} \\
\hline & STAC & STDC & FGAC & FGDC & FGGAC & FGGDC \\
\hline \multicolumn{7}{|c|}{ 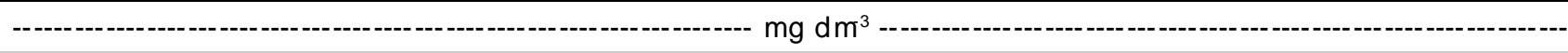 } \\
\hline & \multicolumn{6}{|c|}{ Antes do terceiro plantio } \\
\hline 50 & 9,00 & 11,00 & 10,33 & 7,67 & 8,33 & 8,00 \\
\hline 100 & 13,67 & 17,33 & 18,67 & 12,00 & 13,33 & 15,33 \\
\hline \multirow[t]{2}{*}{200} & 28,33 & 32,33 & 25,00 & 20,00 & 21,67 & 23,67 \\
\hline & \multicolumn{6}{|c|}{ Depois do último corte } \\
\hline 50 & 9,67 & 10,67 & 9,33 & 10,67 & 9,00 & 9,67 \\
\hline 100 & 13,67 & 15,67 & 22,00 & 21,00 & 14,67 & 17,33 \\
\hline 200 & 26,00 & 27,67 & 40,67 & 31,33 & 33,00 & 31,33 \\
\hline
\end{tabular}

(STAC: super triplo antes da calagem; STDC: super triplo depois da calagem; FGAC: Fosfato de Gafsa antes da calagem; FGDC: Fosfato de Gafsa depois da calagem; FGGAC: Fosfato de Gafsa com gesso antes da calagem; FGGDC: Fosfato de Gafsa com gesso depois da calagem).

CV $($ parcela $)=5,00 \%$. CV $($ subparcela $)=5,12 \%$ 
TABELA 11 - Contrastes ortogonais para comparação do teor de $P$ no solo entre os tratamentos com fontes de fósforo dentro de cada período de amostragem do solo, no terceiro plantio.

\begin{tabular}{llcc}
\hline & \multicolumn{1}{c}{ Contrastes } & \multicolumn{2}{c}{ Momento de amostragem } \\
\cline { 3 - 4 } & & Antes do plantio & Após o último corte \\
\hline № & & ---- Valor do contraste -------- \\
26 & 2STAC + 2STDC - FGAC - FGDC - FGGAC - FGGDC & $0,64^{*}$ & $-0,51^{*}$ \\
27 & STAC - STDC & $-0,17$ & $-0,10$ \\
28 & FGAC + FGDC - FGGAC - FGGDC & 0,08 & $0,30^{*}$ \\
29 & FGAC - FGDC & $0,32^{*}$ & 0,05 \\
30 & FGGAC - FGGDC & $-0,06$ & $-0,07$ \\
\hline
\end{tabular}

(STAC: super triplo antes da calagem; STDC: super triplo depois da calagem; FGAC: Fosfato de Gafsa antes da calagem; FGDC: Fosfato de Gafsa depois da calagem; FGGAC: Fosfato de Gafsa com gesso antes da calagem; FGGDC: Fosfato de Gafsa com gesso depois da calagem).

${ }^{*}$ significativo a $5 \%$.

TABELA 12 - Teor de P no solo antes do terceiro plantio e após o último corte da alfafa, em função dos tratamentos com fontes de P. (Média de três repetições e três doses de P).

\begin{tabular}{lcccccc}
\hline & \multicolumn{7}{c}{ Tratamentos com fontes de P } \\
\cline { 2 - 6 } Momento de amostragem & STAC & STDC & FGAC & FGDC & FGGAC & FGGDC \\
\hline & $15,07 \mathrm{a}$ & $17,91 \mathrm{a}$ & $16,76 \mathrm{~b}$ & $12,17 \mathrm{~b}$ & $13,30 \mathrm{~b}$ & $14,11 \mathrm{~b}$ \\
Antes do plantio & $15,06 \mathrm{a}$ & $16,57 \mathrm{a}$ & $19,78 \mathrm{a}$ & $18,90 \mathrm{a}$ & $16,07 \mathrm{a}$ & $17,28 \mathrm{a}$ \\
\hline
\end{tabular}

(STAC: super triplo antes da calagem; STDC: super triplo depois da calagem; FGAC: Fosfato de Gafsa antes da calagem; FGDC: Fosfato de Gafsa depois da calagem; FGGAC: Fosfato de Gafsa com gesso antes da calagem; FGGDC: Fosfato de Gafsa com gesso depois da calagem).

*médias seguidas por letras distintas, numa mesma coluna, diferem a $5 \%$ pelo teste de Tukey.

$\mathrm{CV}=5,12 \%$.

Não houve efeito $(P>0,05)$ do momento da calagem para o ST nos dois períodos de amostragens (TABELA 10 e contraste 27 da TABELA 11).

Ocorreu maior $(P<0,05)$ concentração de $P$ no solo somente no tratamento FGAC comparado ao FGDC, no primeiro período de amostragem do solo (TABELA 10 e contraste 29 da TABELA 11). Esse resultado parece contraditório uma vez que isso não refletiu em maior produção de MS da parte aérea com a utilização do FGAC em relação ao FGDC (TABELA 2 e contrastes 9, 14 e 19 da TABELA 3). Houve, ao contrário, maior $(\mathrm{P}<0,05)$ produção de MS da parte aérea com o uso do FGDC comparado ao FGAC, na dose de $50 \mathrm{mg} \mathrm{P} \mathrm{dm}^{-3}$ no terceiro corte do terceiro plantio (TABELA 2 e contraste 19 da TABELA 3).

Não houve efeito $(P>0,05)$ do gesso adicionado ao $F G$ sobre a concentração de $P$ no solo quando realizaram-se amostragens do solo antes do terceiro plantio (TABELA 10 e contraste 28 da TABELA 11). Dessa maneira pode-se concluir que o teor de $\mathrm{P}$ no solo, com a utilização do gesso associado ao $F G$, não foi o responsável por maiores $(P<0,05)$ produções de $M S$ da parte aérea nos tratamentos FGGAC e FGGDC em relação aos tratamentos sem gesso na dose de $200 \mathrm{mg}$ $P \mathrm{dm}^{-3}$ (TABELA 2 e contraste 8 da TABELA 3). Assim, pode-se inferir que a associação do gesso com o FG não provoca, em vaso, maior disponibilidade de $\mathrm{P}$ no solo.

No entanto, o teor de $P$ no solo apresentouse maior $(P<0,05)$ sem 0 uso do gesso (FGAC e
FGDC) do que com sua aplicação (FGGAC e FGGDC), depois do último corte (TABELA 10 e contraste 28 da TABELA 11). Isso pode ser devido a maior retirada do $P$ do solo nos tratamento FGGAC e FGGDC, uma vez que, em geral, esses tratamentos tenderam a apresentar maiores produções de MS do que o FGAC e FGDC, no terceiro plantio (TABELA 2). Essas observações concordam com as de Pons, citado por Macedo et al. (1985), trabalhando com alfafa cultivada em solo classificado como Latossolo Bruno Distrófico.

\section{CONCLUSÕES}

Inicialmente o superfosfato triplo proporciona maiores produções de matéria seca que o fosfato de Gafsa associado ou não ao gesso. O fosfato de Gafsa é uma eficiente fonte de $P$ após a incubação de cinco meses. Na implantação de alfafa o calcário deve ser aplicado antes do super triplo. A aplicação da calagem antes ou após o fosfato de Gafsa não influencia a solubilização desse fosfato. A adição de gesso ao fosfato de Gafsa tende a aumentar a produção de matéria seca, como também melhora $0 \mathrm{pH}$ do solo e a produção do sistema radicular. A alfafa não se estabelece em LATOSSOLO VERMELHO-AMARELO Alumínico com teor de $\mathrm{P}$ de $3 \mathrm{mg} \mathrm{P} \mathrm{dm}^{-3}$. Teores de $\mathrm{P}$ no solo acima de $30 \mathrm{mg} \mathrm{P} \mathrm{dm}^{-3}$ são suficientes, em vaso, para elevadas produções de alfafa. 


\section{AGRADECIMENTO}

À FAPESP pelo financiamento da pesquisa.

\section{REFERÊNCIAS BIBLIOGRÁFICAS}

CHU, C.R.; MOSCHLER, W.W.; THOMAS, G.W. Rock phosphate transformations in acid soils. Soil Science Society of America Proceedings, v.26, p.476-478, 1962.

GOEDERT, W.J.; LOBATO, E. Avaliação agronômica de fosfatos em solo de cerrado. Revista Brasileira de Ciência do Solo, v.8, p.97-102, 1984

GOEDERT, W.J.; SOUSA, D.M.G. Uso eficiênte de fertilizantes fosfatados. In: SIMPÓSIO SOBRE FERTILIZANTES NA AGRICULTURA BRASILEIRA, Brasília, 1984. Anais. Brasília: EMBRAPA, 1984. p.206-255. (Documentos,14).

HANAFI, M.M.; SYERS, J.K.; BOLAN, N.S. Effect of lime on the dissolution of two phosphate rocks in acid soil. Journal of the Science of Food and Agriculture, v.60, p.155-164, 1992.

HEICHEL, G.H.; DELANEY, R.H.; CRALLE, H.T. Carbon assimilation, partitioning and utilization. In: HANSON, C.H. (Ed.) Alfalfa and alfalfa improvement. Madison: ASA, CSSA, SSSA, 1988 . cap.6, p.195-222

HONDA, C.S.; HONDA, A.M. Cultura da alfafa. Cambara: IARA, 1990. 245p.

KABATA-PENDIAS, A.; PENDIAS, $H$. Trace elements in soils and plants. 3.ed. Boca Roton: CRC Press, 1985. 315p.

MACEDO, W.; GONÇALVES J.O.N.; GIRARDI-DEIRO, A.M. Melhoramento de pastagem natural com fosfatos e introdução de leguminosas em solo da fronteira oeste do Rio Grande do Sul. Revista Brasileira de Ciência do Solo, v.9, p.231-235, 1985.

MAGALHÃES, J.C.A.J.; MELLO, F.A.F.; THOMAZI, M.D. Avaliação agronômica de fosfatos naturais com ênfase para solos sob vegetação de cerrado: I. Revista de Agricultura, v.62, p.61-89, 1987.

MOREIRA, A. Efeito de fontes e doses de fósforo na alfafa (Medicago sativa L.) e centrosema (Centrosema pubens Benth.) e avaliação de extratores. Piracicaba, 1997. 107p. Dissertação (Mestrado) - Escola Superior de Agricultura "Luiz de Queiroz", Universidade de São Paulo.

MUGWIRA, L.M.; HAQUE, I. Screening forage and browse legumes germplasm to nutrient stress I: tolerance of Medicago sativa $\mathrm{L}$. To aluminum and low phosphorus in soils and nutrient solutions. Journal of Plant Nutrition, v.16, p.17-35, 1993.
NOVAIS, R.F.; NEVES, J.C.L.; BARRO, N.F. Curso de fertilidade do solo: módulo 7 - fósforo. Brasília: ABEAS, 1990. 132p.

RAIJ, B. van Gesso agrícola na melhoria do ambiente radicular no subsolo. São Paulo: ANDA, 1988. 88p.

RAIJ, B. van Fertilidade do solo e adubação. São Paulo: Ceres, 1991. 343p.

RAIJ, B. van; ROSAND, P.C.; LOBATO, E. Adubação fosfatada no Brasil. Planaltina: EMBRAPA, CPAC, 1992. 326p. (Documentos, 21).

RAIJ, B. van; QUAGGIO, J.A.; SILVA, N.M. Extraction of phosphorus, potassium, calcium and magnesium from soil by na ion-exchange resin procedure. Communications in Soil Science and Plant Analysis, v. 17, p.547-566, 1986.

RANDO, E.M. Desenvolvimento da alfafa (Medicago sativa L.) em diferentes níveis de $\mathrm{pH}$, potássio e enxofre no solo/tese. Piracicaba, 1992. 220p. Tese (Doutorado) - Escola Superior de Agricultura "Luiz de Queiroz", Universidade de São Paulo.

RECHCIGL, J.E.; EDMISTEN, K.L.; WOLF, D.D.; RENEAU, R.B. Response of alfalfa grown on acid soil to different chemical amendments. Agronomy Journal, v.80, p.515-518, 1988.

RHYKERD, C.L.; OVERDAHL, C.J. Nutrition and fertilizer use. In: HANSON, A.A.; CARENCE, $H$. Alfafa science and technology. Madison: ASA, 1972. cap.8, p.437-465.

SARRIÉS, G.A.; ALVES, M.C.; OLIVEIRA, J.C.V. Sanest. Piracicaba: USP, ESALQ, CIAGRI, 1993. 57p. (Série Didática, 6).

SMITH, G.S.; EDMEADES, D.C.; UPSDELL, M. Manganese status of New Zealand pastures 1. Toxicity in ryegrass, white clover, and lucerne. New Zealand Journal of Agricultural Research, v.26, p.215-221, 1983.

SMYTH, T.J.; SANCHEZ, P.A. Effects of lime, and phosphorus apllications to an oxisol on phosphorus sorption and ion retention. Soil Science Society of America Journal, v.44, p.500-505, 1980.

SOUSA, D.M.G.; RITCHEY, K.D. Uso de gesso no solo de cerrado. In: SEMINÁRIO SOBRE O USO DE FOSFOGESSO NA AGRICULTURA, 1., Brasília, 1985. Anais. Brasília: EMBRAPA, 1985. p.119-144.

YUPANQUI, F.F.R. Nutrição fosfatada e fotossíntese no sistema simbiótico Medicago sativa - Rhizobium meliloti em solos com diferentes disponibilidades de fósforo. Viçosa, 1997. 124p. Tese (Doutorado) - Universidade Federal de Viçosa.

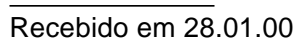

